\title{
Highly oscillatory quadrature: The story so far
}

\author{
A. Iserles ${ }^{1}$, S.P. Nørsett ${ }^{2}$, and S. Olver ${ }^{3}$
}

1 Department of Applied Mathematics and Theoretical Physics, Centre for Mathematical Sciences, University of Cambridge, Wilberforce Road, Cambridge CB3 0WA, United Kingdom, A.Iserles@damtp.cam.ac.uk

2 Department of Mathematical Sciences, Norwegian University of Science and Technology, 7491 Trondheim, Norway, S.P.Norsett@math.ntnu.no

3 Department of Applied Mathematics and Theoretical Physics, Centre for Mathematical Sciences, University of Cambridge, Wilberforce Road, Cambridge CB3 0WA, United Kingdom, S.01ver@damtp.cam.ac.uk

Summary. The last few years have witnessed substantive developments in the computation of highly oscillatory integrals in one or more dimensions. The availability of new asymptotic expansions and a Stokes-type theorem allow for a comprehensive analysis of a number of old (although enhanced) and new quadrature techniques: the asymptotic, Filon-type and Levin-type methods. All these methods share the surprising property that their accuracy increases with growing oscillation. These developments are described in a unified fashion, taking the multivariate integral $\int_{\Omega} f(\boldsymbol{x}) \mathrm{e}^{\mathrm{i} \omega g(\boldsymbol{x})} \mathrm{d} V$ as our point of departure.

\section{The challenge of high oscillation}

Rapid oscillation is ubiquitous in applications and is, by common consent, considered a 'difficult' problem. Indeed, the standard technique of dealing with high oscillation is to make it disappear by sampling the signal sufficiently frequently, and this typically leads to prohibitive cost.

The subject of this article is a review of recent work on the computation of integrals of the form

$$
I[f, \Omega]=\int_{\Omega} f(\boldsymbol{x}) \mathrm{e}^{\mathrm{i} \omega g(\boldsymbol{x})} \mathrm{d} V,
$$

where $\Omega \subset \mathbb{R}^{n}$ is a bounded open domain with piecewise-smooth boundary, while $f$ and the oscillator $g$ are smooth. We assume in (1) that $\omega \in \mathbb{R}$ is large in modulus, hence $I[f, \Omega]$ oscillates rapidly as a function of $\omega$.

A natural technique to compute (1) in a univariate setting is Gaussian quadrature. Yet, a moment's reflection clarifies that it is likely to be absolutely useless unless $|\omega|$ is small. Classical quadrature (with a trivial weight function) is just an exact integration of a polynomial interpolation of the integrand. 
However, if the integrand oscillates rapidly, and unless we use an astronomical number of function evaluations, polynomial interpolation is useless! This is vividly demonstrated in Fig. 1. We have computed

$\int_{-1}^{1} \cos x \mathrm{e}^{\mathrm{i} \omega x^{2}} \mathrm{~d} x=-\frac{\pi^{\frac{1}{2}}}{2(-\mathrm{i} \omega)^{\frac{1}{2}}} \exp \left(\frac{1}{4} \frac{1}{\mathrm{i} \omega}\right)\left[\operatorname{erf}\left(\mathrm{i} \frac{\omega+\frac{1}{2}}{(-\mathrm{i} \omega)^{\frac{1}{2}}}\right)+\operatorname{erf}\left(\mathrm{i} \frac{\omega-\frac{1}{2}}{(-\mathrm{i} \omega)^{\frac{1}{2}}}\right)\right]$

by Gaussian quadrature with different number of points. The figure displays the absolute value of the error as a function of $\omega \in[0,100]$. Note that, as long as $\omega$ is small, everything is fine, but as soon as $\omega$ is large in comparison with the number of quadrature points and high oscillation sets in, the error becomes $\mathcal{O}(1)$. As a matter of fact, given that $I[f] \sim \mathcal{O}\left(\omega^{-\frac{1}{2}}\right)$, the trivial approximation $I[f] \approx 0$ is far superior to Gaussian quadrature with 30 points!

Yet, efficient and cheap quadrature of (1) is perfectly possible. Indeed, once we understand the mathematical mechanism underlying (1), we can compute it to high precision with minimal effort and, perhaps paradoxically, the quality of approximation increases with $\omega$.
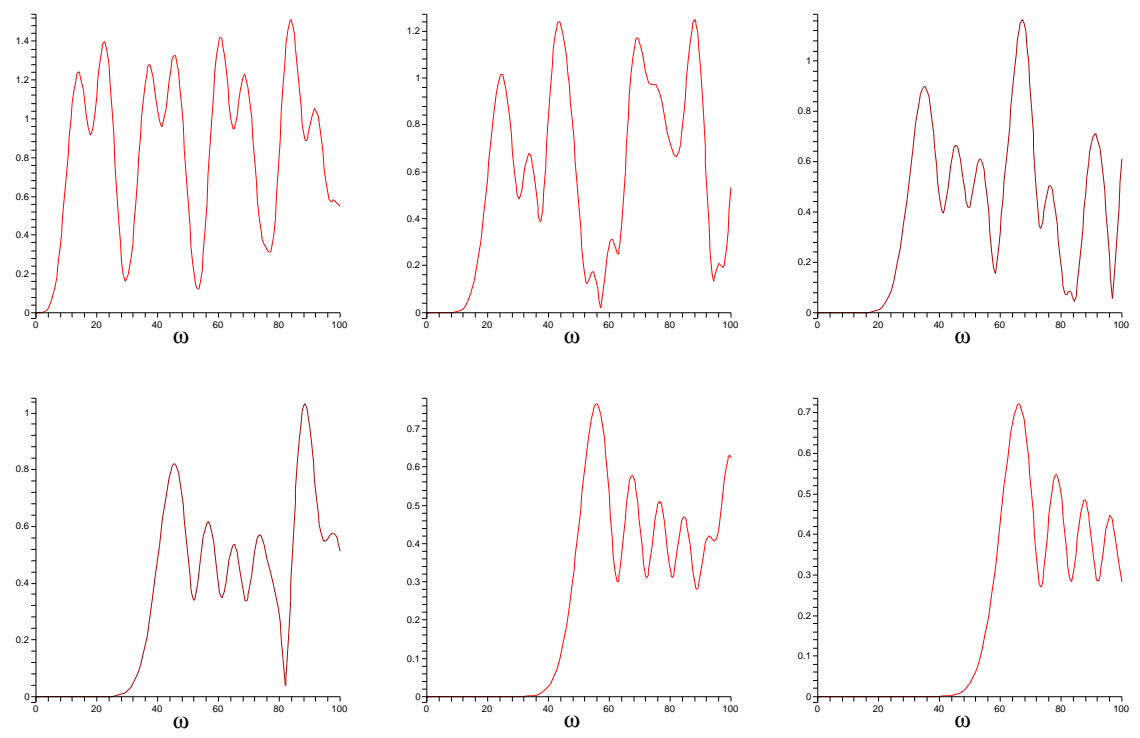

Fig. 1. Error in Gaussian quadrature with $\Omega=(-1,1), f(x)=\cos x, g(x)$ and $\nu$ points. Here $\nu$ increases by increments of five, from 5 to 30 .

This article collates a sequence of papers by the authors into unified narrative. In particular, we revisit here the work of (Iserles \& Nørsett 2005a, Iserles \& Nørsett 2006, Olver 2005a) and (Olver 2005b), to which the reader is re- 
ferred for technical details, more comprehensive exposition and a wealth of further numerical examples.

The conventional organising principle of quadrature is a Taylor expansion. Once the integrand oscillates rapidly, a Taylor expansion converges very slowly indeed and is, to all intents and purposes, useless. Instead, we need to exploit an asymptotic expansion in negative powers of $\omega$. In Section 2 we present an asymptotic expansion of (1) in the case when the oscillator $g$ has no critical points: $\boldsymbol{\nabla} g(\boldsymbol{x}) \neq \mathbf{0}$ for all $\boldsymbol{x} \in \mathrm{cl} \Omega$ and subject to the nonresonance condition: $\boldsymbol{\nabla} g(\boldsymbol{x})$ is not allowed to be normal to the boundary $\partial \Omega$ for any $\boldsymbol{x} \in \partial \Omega$.

The availability of an asymptotic expansion allows us to design and analyse effective quadrature methods, and this is the subject of Section 3. We single out for consideration three general techniques: asymptotic methods, consisting of a truncation of the asymptotic expansion of Section 2, Filon-type methods, which interpolate just $f(\boldsymbol{x})$, rather than the entire integral (Filon 1928), and Levin-type methods, which collocate the integrand (Levin 1982).

In Section 4 we consider the case when critical points are allowed. A comprehensive theory exists, as things stand, only in one dimension, hence we focus on $g:[a, b] \rightarrow \mathbb{R}$ and study the case of $g^{\prime}(\xi)=0$ for some $\xi \in[a, b]$, $g^{\prime} \neq 0$ for $[a, b] \backslash\{\xi\}$. (Obviously, we are allowed, without loss of generality, to assume the existence of just one critical point: otherwise we integrate in a finite number of subintervals.) An asymptotic expansion in the presence of a critical point presents us with new challenges. In principle, we could have used here the standard technique of stationary phase (Olver 1974, Stein 1993), except that it is not equal to our task. We present an alternative that leads to an explicit and workable expansion. It is subsequently used to design asymptotic and Filon-type methods: unfortunately, Levin-type methods are not available in this setting.

The purpose of the final section is the sketch gaps in the theory and comment on ongoing challenges and developments. Moreover, we describe there briefly the recent method of Huybrechs \& Vandewalle (2005), as well as the work in progress in Cambridge and Trondheim.

Quadrature of (1) represents but one problem in the wide range of issues originating in high oscillation. Quite clearly, a more significant challenge is to solve highly oscillatory differential equations. It is thus of interest to mention that the availability of efficient highly oscillatory quadrature is critical to a number of contemporary methods for ordinary differential equations that exhibit rapid oscillation (Degani \& Schiff 2003, Iserles 2002, Iserles 2004, Lorenz, Jahnke \& Lubich 2005).

\section{Asymptotic expansion in the absence of critical points}

We restrict our analysis to $\mathbb{R}^{2}$, directing the reader to (Iserles \& Nørsett 2006) for the general case. Let first $\Omega=\mathcal{S}_{2}$, the triangle with vertices at $(0,0),(1,0)$ and $(0,1)$. The nonresonance condition is thus 


$$
\begin{aligned}
& g_{y}(x, 0) \neq 0, \quad x \in[0,1], \quad g_{x}(0, y) \neq 0, \quad y \in[0,1], \\
& g_{x}(x, y)-g_{y}(x, y) \neq 0, \quad x, y \geq 0, \quad x+y \in[0,1] .
\end{aligned}
$$

Integrating by parts in the inner integral,

$$
\begin{aligned}
I\left[g_{x}^{2} f, \mathcal{S}_{2}\right]= & \int_{0}^{1} \int_{0}^{1-y} g_{x}^{2}(x, y) f(x, y) \mathrm{e}^{\mathrm{i} \omega g(x, y)} \mathrm{d} x \mathrm{~d} y \\
= & \frac{1}{\mathrm{i} \omega} \int_{0}^{1} g_{x}(1-y, y) f(1-y, y) \mathrm{e}^{\mathrm{i} \omega g(1-y, y)} \mathrm{d} y \\
& -\frac{1}{\mathrm{i} \omega} \int_{0}^{1} g_{x}(0, y) f(0, y) \mathrm{e}^{\mathrm{i} \omega g(0, y)} \mathrm{d} y-\frac{1}{\mathrm{i} \omega} I\left[\frac{\partial}{\partial x}\left(g_{x} f\right), \mathcal{S}_{2}\right] \\
= & \frac{1}{\mathrm{i} \omega} \int_{0}^{1} g_{x}(x, 1-x) f(x, 1-x) \mathrm{e}^{\mathrm{i} \omega g(x, 1-x)} \mathrm{d} x \\
& -\frac{1}{\mathrm{i} \omega} \int_{0}^{1} g_{x}(0, y) f(0, y) \mathrm{e}^{\mathrm{i} \omega g(0, y)} \mathrm{d} y-\frac{1}{\mathrm{i} \omega} I\left[\frac{\partial}{\partial x}\left(g_{x} f\right), \mathcal{S}_{2}\right] .
\end{aligned}
$$

By the same token,

$$
\begin{aligned}
I\left[g_{y}^{2} f, \mathcal{S}_{2}\right]= & \frac{1}{\mathrm{i} \omega} \int_{0}^{1} g_{y}(x, 1-x) f(x, 1-x) \mathrm{e}^{\mathrm{i} \omega g(x, 1-x)} \mathrm{d} x \\
& -\frac{1}{\mathrm{i} \omega} \int_{0}^{1} g_{y}(x, 0) f(x, 0) \mathrm{e}^{\mathrm{i} \omega g(x, 0)} \mathrm{d} y-\frac{1}{\mathrm{i} \omega} I\left[\frac{\partial}{\partial y}\left(g_{y} f\right), \mathcal{S}_{2}\right] .
\end{aligned}
$$

Adding up, we have

$$
I\left[\|\nabla g\|^{2} f, \mathcal{S}_{2}\right]=\frac{1}{\mathrm{i} \omega}\left(M_{1}+M_{2}+M_{3}\right)-\frac{1}{\mathrm{i} \omega} I\left[\nabla^{\top}(f \boldsymbol{\nabla} g), \mathcal{S}_{2}\right],
$$

where

$$
\begin{aligned}
& M_{1}=\int_{0}^{1} f(x, 0)\left[\boldsymbol{n}_{1}^{\top} \boldsymbol{\nabla} g(x, 0)\right] \mathrm{e}^{\mathrm{i} \omega g(x, 0)} \mathrm{d} x, \\
& M_{2}=\sqrt{2} \int_{0}^{1} f(x, 1-x)\left[\boldsymbol{n}_{2}^{\top} \boldsymbol{\nabla} g(x, 1-x)\right] \mathrm{e}^{\mathrm{i} \omega g(x, 1-x)} \mathrm{d} x, \\
& M_{3}=\int_{0}^{1} f(0, y)\left[\boldsymbol{n}_{3}^{\top} \boldsymbol{g}(0, y)\right] \mathrm{e}^{\mathrm{i} \omega g(0, y)} \mathrm{d} y .
\end{aligned}
$$

Here

$$
\boldsymbol{n}_{1}=\left[\begin{array}{c}
0 \\
-1
\end{array}\right], \quad \boldsymbol{n}_{2}=\left[\begin{array}{c}
\frac{\sqrt{2}}{2} \\
\frac{\sqrt{2}}{2}
\end{array}\right], \quad \boldsymbol{n}_{3}=\left[\begin{array}{c}
-1 \\
0
\end{array}\right]
$$

are outward unit normals at the edges of $\mathcal{S}_{2}$.

Since $\boldsymbol{\nabla} g(x, y) \neq \mathbf{0}$ in $\mathrm{cl} \mathcal{S}_{2}$, we may replace above $f$ by $f /\|\nabla g\|^{2}$ without any danger of dividing by zero. The outcome is 


$$
\begin{aligned}
I\left[f, \mathcal{S}_{2}\right]= & \frac{1}{\mathrm{i} \omega} \int_{\partial \mathcal{S}_{2}} \boldsymbol{n}^{\top}(\boldsymbol{x}) \boldsymbol{\nabla} g(\boldsymbol{x}) \frac{f(\boldsymbol{x})}{\|\boldsymbol{\nabla} g(\boldsymbol{x})\|^{2}} \mathrm{e}^{\mathrm{i} \omega g(\boldsymbol{x})} \mathrm{d} S \\
& -\frac{1}{\mathrm{i} \omega} I\left[\boldsymbol{\nabla}^{\top}\left(\frac{f}{\|\boldsymbol{\nabla} g\|^{2}} \boldsymbol{\nabla} g\right), \mathcal{S}_{2}\right] .
\end{aligned}
$$

Extending this technique to $\mathbb{R}^{n}$, it is possible to prove that (2) remains true once we replace $\mathcal{S}_{2}$ by $\mathcal{S}_{n} \subset \mathbb{R}^{n}$, the regular simplex with vertices at $\mathbf{0}$ and $\boldsymbol{e}_{1}, \ldots, \boldsymbol{e}_{n}$.

Let

$$
f_{0}(\boldsymbol{x})=f(\boldsymbol{x}), \quad f_{m}=\boldsymbol{\nabla}^{\top}\left[\frac{f_{m-1}(\boldsymbol{x})}{\|\boldsymbol{\nabla} g(\boldsymbol{x})\|^{2}} \boldsymbol{\nabla} g(\boldsymbol{x})\right], \quad m \in \mathbb{N} .
$$

We deduce from (2) (extended to $\mathbb{R}^{n}$ ) that

$I\left[f_{m}, \mathcal{S}_{n}\right]=\frac{1}{\mathrm{i} \omega} \int_{\partial \mathcal{S}_{n}} \boldsymbol{n}^{\top} \boldsymbol{\nabla} g(\boldsymbol{x}) \frac{f_{m}(\boldsymbol{x})}{\|\boldsymbol{\nabla} g(\boldsymbol{x})\|^{2}} \mathrm{e}^{\mathrm{i} \omega g(\boldsymbol{x})} \mathrm{d} S-\frac{1}{\mathrm{i} \omega} I\left[f_{m+1}, \mathcal{S}_{n}\right], \quad m \in \mathbb{Z}_{+}$.

Finally, we iterate the above expression to obtain a Stokes-type formula, expressing $I\left[f, \mathcal{S}_{n}\right]$ as an asymptotic expansion on the boundary of the simplex,

$$
I\left[f, \mathcal{S}_{n}\right] \sim-\sum_{m=0}^{\infty} \frac{1}{(-\mathrm{i} \omega)^{m+1}} \int_{\partial \mathcal{S}_{n}} \boldsymbol{n}^{\top} \boldsymbol{\nabla} g(\boldsymbol{x}) \frac{f_{m}(\boldsymbol{x})}{\|\boldsymbol{\nabla} g(\boldsymbol{x})\|^{2}} \mathrm{e}^{\mathrm{i} \omega g(\boldsymbol{x})} \mathrm{d} S .
$$

We wish to highlight four important issues. Firstly, a trivial inductive proof confirms that each $f_{m}$ can be expressed as a linear combination of $f$ and the first $m$ directional derivatives (altogether, $\left(\begin{array}{c}n+m+1 \\ m\end{array}\right)$ quantities), with coefficients that depend on the oscillator $g$ and its derivatives.

Secondly, the simplest (and most useful) special case is $n=1$, whence (3) reduces to

$$
I[f,(0,1)] \sim-\sum_{m=0}^{\infty} \frac{1}{(-\mathrm{i} \omega)^{m+1}}\left[\frac{\mathrm{e}^{\mathrm{i} \omega g(1)}}{g^{\prime}(1)} f_{m}(1)-\frac{\mathrm{e}^{\mathrm{i} \omega g(0)}}{g^{\prime}(0)} f_{m}(0)\right] .
$$

Thirdly, using an affine transformation, we can map $\mathcal{S}_{n}$ to an arbitrary simplex in $\mathbb{R}^{n}$. Applying an identical transformation to (3), we deduce that it is valid for $I[f, \mathcal{S}]$, where $\mathcal{S} \subset \mathbb{R}^{n}$ is any simplex.

Fourthly, the boundary of $\mathcal{S}$ is itself composed of $n+1$ simplices in $\mathbb{R}^{n-1}$. Because of the nonresonance condition, the gradient of the oscillator does not vanish in any of these simplices and we can apply (3) therein: this expresses $I[f, \mathcal{S}]$ as an asymptotic expansion over $(n-2)$-dimensional simplices. We continue with this procedure until we reach 0-dimensional simplices: the $n+1$ vertices of the original simplex. Bearing in mind our first observation, we thus deduce that

$$
I[f, \mathcal{S}] \sim \sum_{m=0}^{\infty} \frac{1}{(-\mathrm{i} \omega)^{m+n}} \Theta_{m}[f]
$$


where each $\Theta_{m}$ is a linear functional which depends on $\partial^{|\boldsymbol{i}|} f / \partial \boldsymbol{x}^{i},|\boldsymbol{i}| \leq m$, at the $n+1$ vertices of $\mathcal{S}$. Note that $I[f, \mathcal{S}]=\mathcal{O}\left(\omega^{-n}\right)$.

In general, the functionals $\Theta_{m}$ are fairly complicated, the univariate case (4) being an exception. However, it is the existence of (5), rather than its exact form, which render possible the design of efficient quadrature methods in the next section.

Let $\Omega \subset \mathbb{R}^{n}$ be a polytope, a bounded (open) domain with piecewise-linear boundary. (Note that $\Omega$ need be neither convex nor even simply connected.) We may then tessellate $\Omega$ with simplices $\Omega_{1}, \Omega_{2}, \ldots, \Omega_{r} \in \mathbb{R}^{n}$, therefore

$$
I[f, \Omega]=\sum_{k=1}^{r} I\left[f, \Omega_{r}\right] .
$$

A simplicial complex is a collection $\mathcal{C}$ of simplices in $\mathbb{R}^{n}$ such that every face of $\Phi \in \mathcal{C}$ is also in $\mathcal{C}$ and if $\Phi_{1} \cap \Phi_{2} \neq \emptyset$ for $\Phi_{1}, \Phi_{2} \in \mathcal{C}$ then $\Phi_{1} \cap$ $\Phi_{2}$ is a face of both $\Phi_{1}$ and $\Phi_{2}$ (Munkres 1991). We may always choose a tessellation composed of all $n$-dimensional simplices in a simplicial complex. In finite-element terminology, this corresponds to a tessellation without 'hanging nodes'.

Assume that the nonresonance condition condition holds for the oscillator $g$. We may always choose a simplicial complex so that the nonresonance condition is valid in each $\Omega_{k}$, otherwise we vary the internal nodes. Clearly, once we can expand asymptotically each $I\left[f, \Omega_{k}\right]$, we may use (6) to expand $I[f, \Omega]$. Bearing in mind (5), this means that the entire information needed to construct such an expansion is the values of $f$ and its derivatives at the vertices of the $\Omega_{k}$ s. However, a moment's reflection clarifies that only the original vertices of $\Omega$ may influence the expansion: the internal vertices are arbitrary, since there is an infinity of simplicial complexes consistent with the nonresonance condition. In other words, because of our construction of the tessellation via a simplicial complex, the contributions from neighbouring simplices cancel at internal vertices and each $\Theta_{m}$ depends on $f$ and its derivatives at the original vertices of $\Omega$.

\section{Asymptotic, Filon and Levin methods}

\subsection{Asymptotic methods}

The simplest and most natural means of approximating (1) consists of a truncation of the asymptotic expansion (5) (replacing $\mathcal{S}$ by a polytope $\Omega$ ). This results in the asymptotic method

$$
Q_{s}^{\mathrm{A}}[f, \Omega]=\sum_{m=0}^{s-1} \frac{1}{(-\mathrm{i} \omega)^{m+n}} \Theta_{m}[f],
$$

bearing an asymptotic error of 


$$
Q_{s}^{\mathrm{A}}[f, \Omega]-I[f, \Omega] \sim \mathcal{O}\left(\omega^{-n-s}\right), \quad|\omega| \gg 1 .
$$

We say that $Q_{s}^{\mathrm{A}}$ is of an asymptotic order $s+n$.

Asymptotic quadrature is particularly straightforward in a single dimension, since then its coefficients are readily provided explicitly by an affine mapping of (4) from $(0,1)$ to an arbitrary bounded real interval.

In Fig. 2 we have plotted the absolute value of the error once

$$
\int_{-1}^{1} x \sin x \mathrm{e}^{\mathrm{i} \omega\left(x+\frac{1}{4} x^{2}\right)} \mathrm{d} x
$$

is approximated by $Q_{s}^{\mathrm{A}}$ with $s=1$ and $s=2$. The error (here and in the sequel) is scaled by $\omega^{p}$, where $p$ is the asymptotic order, otherwise the rate of decay at the plot would have been so rapid as to prevent much useful insight. It is clear that, exactly as predicted by our theory, the error indeed decays as $\psi(\omega) / \omega^{p}$, where $\psi$ is a bounded function.
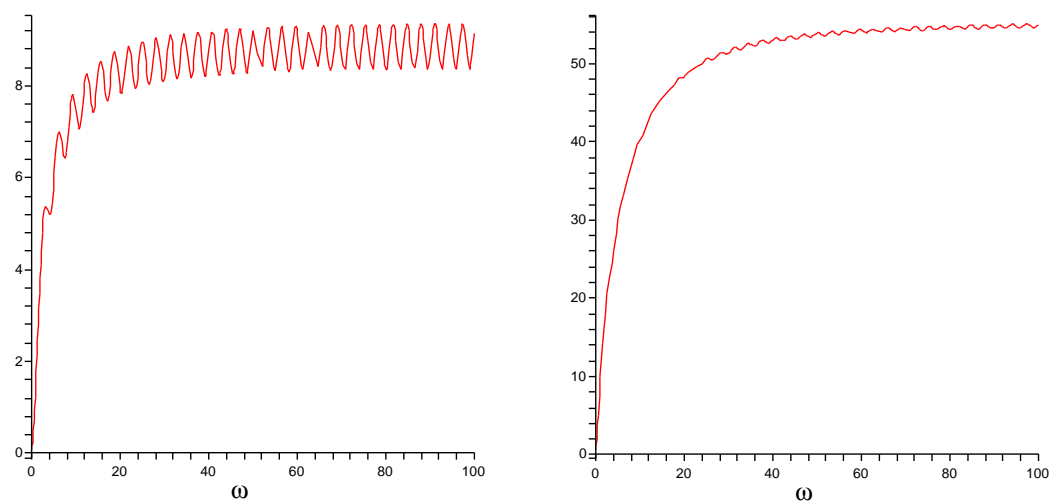

Fig. 2. Error, scaled by $\omega^{p}$, in asymptotic quadrature of asymptotic order $p$ with $\Omega=(-1,1), f(x)=x \sin x, g(x)=x+\frac{1}{4} x^{2}$ and $s=1, p=2$ (on the left) and $s=2$, $p=3$ (on the right).

The coefficients of an asymptotic method are becoming fairly elaborate in $n \geq 2$ dimensions. Thus, for example, for the linear oscillator $g(x, y)=$ $\kappa_{1} x+\kappa_{2} y$ we have

$$
\begin{aligned}
Q_{2}^{\mathrm{A}}\left[f, \mathcal{S}_{2}\right]= & \frac{1}{(-\mathrm{i} \omega)^{2}}\left[\frac{1}{\kappa_{1} \kappa_{2}} f(0,0)+\frac{\mathrm{e}^{\mathrm{i} \kappa_{1} \omega}}{\kappa_{1}\left(\kappa_{1}-\kappa_{2}\right)} f(1,0)-\frac{\mathrm{e}^{\mathrm{i} \kappa_{2} \omega}}{\kappa_{2}\left(\kappa_{1}-\kappa_{2}\right)} f(0,1)\right] \\
& +\frac{1}{(-\mathrm{i} \omega)^{3}}\left\{\left[\frac{1}{\kappa_{1}^{2} \kappa_{2}} f_{x}(0,0)+\frac{1}{\kappa_{1} \kappa_{2}^{2}} f_{y}(0,0)\right]\right. \\
& +\mathrm{e}^{\mathrm{i} \kappa_{1} \omega}\left[\frac{2 \kappa_{1}-\kappa_{2}}{\kappa_{1}^{2}\left(\kappa_{1}-\kappa_{2}\right)^{2}} f_{x}(1,0)-\frac{1}{\kappa_{1}\left(\kappa_{1}-\kappa_{2}\right)^{2}} f_{y}(1,0)\right]
\end{aligned}
$$




$$
\left.+\mathrm{e}^{\mathrm{i} \kappa_{2} \omega}\left[-\frac{1}{\kappa_{2}\left(\kappa_{1}-\kappa_{2}\right)^{2}} f_{x}(0,1)+\frac{-\kappa_{1}+2 \kappa_{2}}{\kappa_{2}^{2}\left(\kappa_{1}-\kappa_{2}\right)^{2}} f_{y}(0,1)\right]\right\} .
$$

Note that all the coefficients are well defined, because of the nonresonance condition.

Fig. 3 exhibits the scaled error of two asymptotic methods, of asymptotic orders 3 and 4 , respectively, in $\mathcal{S}_{2}$. Yet, it is fair to comment that the sheer complexity of the coefficients for general oscillators and polytopes limits the application of (7) mainly to the univariate case. Another important shortcoming of an asymptotic method is that, given $\omega$ and the number of derivatives that we may use, its accuracy, although high, is predetermined. Often we may increase accuracy by using higher derivatives, but even this is not assured, since asymptotic expansions do not converge in the usual sense. Once $\omega$ is fixed, it is entirely possible that $Q_{s}^{\mathrm{A}}$ for some $s \geq 1$ is superior to $Q_{r}^{\mathrm{A}}$ for all $r>s$.
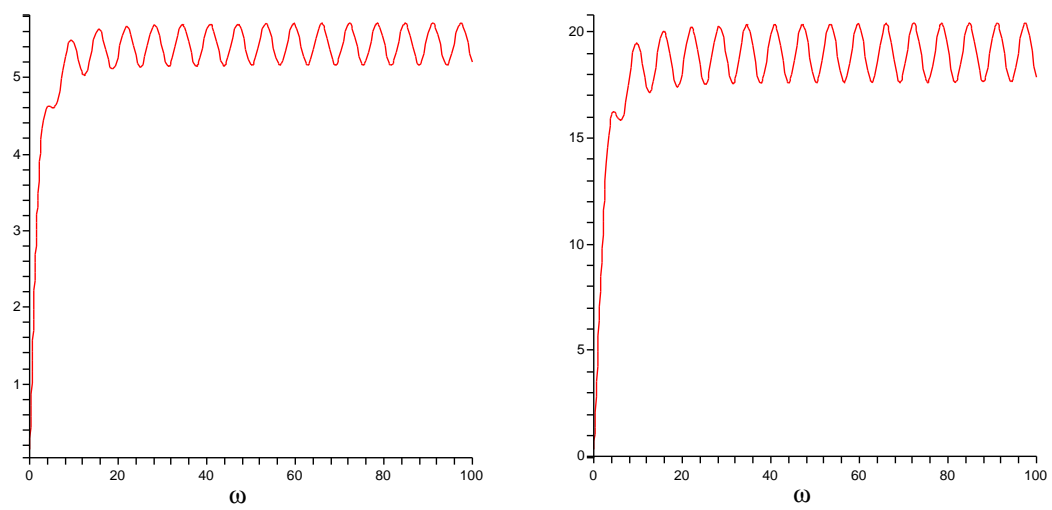

Fig. 3. Scaled error for $Q_{1}^{\mathrm{A}}$ (on the left) and $Q_{2}^{\mathrm{A}}$ (on the right) for $\Omega=\mathcal{S}_{2}, f(x, y)=$ $\mathrm{e}^{x-2 y}$ and $g(x, y)=x+2 y$.

\subsection{Filon-type methods}

Although an asymptotic method (7) is the most obvious consequence of the asymptotic expansion (5), it is by no means the most effective. A more sophisticated use of the asymptotic expansion rapidly leads to far superior, accurate and versatile quadrature schemes.

Let $\varphi$ be an arbitrary smooth function in the closure of the polytope $\Omega \subset \mathbb{R}^{n}$ and suppose that at every vertex $\boldsymbol{v} \in \mathbb{R}^{n}$ of $\Omega$ it is true that

$$
\frac{\partial^{|\boldsymbol{i}|}}{\partial \boldsymbol{x}^{\boldsymbol{i}}} \varphi(\boldsymbol{v})=\frac{\partial^{|\boldsymbol{i}|}}{\partial \boldsymbol{x}^{\boldsymbol{i}}} f(\boldsymbol{v}), \quad 0 \leq|\boldsymbol{i}| \leq s-1 .
$$


It then follows at once from (5) (where, again, we have replaced $\mathcal{S}$ with $\Omega$ ) that

$$
I[\varphi, \Omega]-I[f, \Omega]=I[\varphi-f, \Omega] \sim \mathcal{O}\left(\omega^{-s-n}\right), \quad|\omega| \gg 1 .
$$

This motivates the Filon-type method

$$
Q_{s}^{\mathrm{F}}[f, \Omega]=I[\varphi, \Omega]=\int_{\Omega} \varphi(\boldsymbol{x}) \mathrm{e}^{\mathrm{i} \omega g(\boldsymbol{x})} \mathrm{d} S .
$$

Needless to say, the above is a 'method' only if $I[\varphi, \Omega]$ can be evaluated exactly. In the most obvious case when $\varphi$ is a polynomial, this is equivalent to the explicit computability of relevant moments of the oscillator $g$,

$$
\mu_{\boldsymbol{i}}(\omega)=\int_{\Omega} \boldsymbol{x}^{i} \mathrm{e}^{\mathrm{i} \omega g(\boldsymbol{x})} \mathrm{d} S, \quad \boldsymbol{x}^{i}=x_{1}^{i_{1}} \cdots x_{n}^{i_{n}}, \quad \boldsymbol{i} \in \mathbb{Z}_{+}^{n} .
$$

We will return to this restriction upon the applicability of (8) in the sequel.

It is important to observe that in the 'minimalist' case, when $\varphi$ interpolates only at the vertices of $\Omega,(7)$ and (8) use exactly the same information. The difference in their performance, which is often substantive, is due solely to the different way this information is processed. While the error in (7) is determined by the asymptotic expansion (5) of $f$, the error of (8) follows from an asymptotic expansion of the interpolation error $\varphi-f$. The latter is likely to be smaller.

Historically, Louis Napoleon George Filon (1928) was the first to contemplate this approach in a single dimension, replacing $f$ by a quadratic approximation at the endpoints and the midpoint. This was generalized by Luke (1954) and Flinn (1960), who have considered general univariate interpolatory quadrature in which $\mathrm{e}^{\mathrm{i} \omega g(x)}$ plays the role of a complex-valued weight function. Yet, a thorough qualitative understanding of such methods and an analysis of their asymptotic order (indeed, the very observation that this concept is germane to their understanding) has been presented only recently: in the univariate case in (Iserles \& Nørsett 2005a) and in a multivariate setting in (Iserles \& Nørsett 2006).

In one dimension we construct Filon-type methods similarly to the familiar interpolatory quadrature rules. Thus, we choose nodes $c_{1}<c_{2}<\cdots<c_{\nu}$, where $c_{1}$ and $c_{\nu}$ are the endpoints of $\Omega$, as well as multiplicities $\boldsymbol{m} \in \mathbb{N}^{\nu}$. The function $\varphi$ is the unique Hermite interpolating polynomial of degree $\mathbf{1}^{\top} \boldsymbol{m}-1$ such that

$$
\varphi^{(i)}\left(c_{k}\right)=f^{(i)}\left(c_{k}\right), \quad i=0, \ldots, m_{k}, \quad k=1,2, \ldots, \nu .
$$

This is consistent with (8) with $s=\min \left\{m_{1}, m_{\nu}\right\}$.

Note that, although asymptotic order is assured by interpolation at the endpoints, it is often useful to interpolate also at internal points, since this usually decreases the error. This is demonstrated in Fig. 4, where we revisit the calculation of Fig. 2 using three Filon-type methods. 

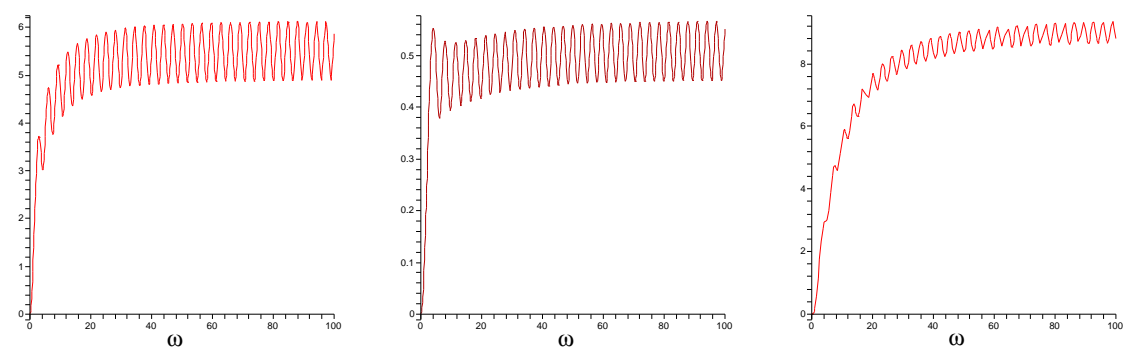

Fig. 4. Scaled error for $Q_{1}^{\mathrm{F}}$ with $\boldsymbol{c}=[-1,1], \boldsymbol{m}=[1,1]$ (on the left), $Q_{1}^{\mathrm{F}}$ with $\boldsymbol{c}=\left[-1,-\frac{3}{4}, \frac{3}{4}, 1\right], \boldsymbol{m}=[1,1,1,1]$ (at the centre) and $Q_{2}^{A}$ with $\boldsymbol{c}=[-1,1], \boldsymbol{m}=[2,2]$ (on the right) for $\Omega=(-1,1), f(x)=x \sin x$ and $g(x)=x+\frac{1}{4} x^{2}$.

Unlike (7), it is fairly straightforward to implement Filon-type methods in a multivariate setting, using standard multivariate approximation theory. The most natural approach is to take a leaf off finite-element theory, tessellate a polytope with simplices (taking care to respect nonresonance) and interpolate in each simplex with suitable polynomials. Note that there is no need to force continuity across edges. In general, the computation of the moments might be problematic, but it is trivial for linear oscillators $g(\boldsymbol{x})=\boldsymbol{\kappa}^{\top} \boldsymbol{x}$.

Fig. 5 displays a bivariate Filon-type quadrature of the integral of Fig. 3. On the left we have used a standard linear interpolation at the vertices. On the right the ten degrees of freedom of a bivariate cubic were quenched by imposing function and first-derivative interpolation at the vertices and simple interpolation at the centroid $\left(\frac{1}{3}, \frac{1}{3}\right)$.
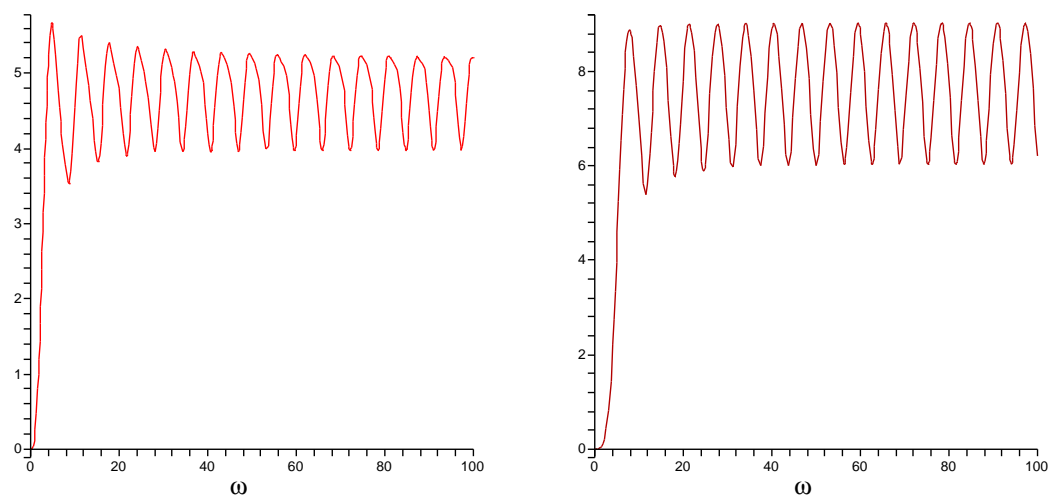

Fig. 5. Scaled error for $Q_{1}^{\mathrm{F}}$ (on the left) and $Q_{2}^{\mathrm{F}}$ (on the right) for $\Omega=\mathcal{S}_{2}, f(x, y)=$ $\mathrm{e}^{x-2 y}$ and $g(x, y)=x+2 y$. 
We mention that it is possible to implement Filon-type methods without the computation of derivatives, using instead finite differences with spacing of $\mathcal{O}\left(\omega^{-1}\right)$ (Iserles \& Nørsett 2005b).

Filon-type methods are highly accurate, affordable and very simple to construct. Yet, there is no escaping their main shortcoming: we must be able to evaluate the moments $\mu_{\boldsymbol{i}}$ of the underlying oscillator. In the next subsection we describe another kind of quadrature methods that use identical information and attain identical asymptotic order without any need to calculate moments.

\subsection{Levin-type methods}

Levin-type methods are quadrature techniques which do not require the computation of moments. Indeed, if $\Omega$ satisfies the nonresonance condition, a Levin-type method can be used to approximate $I[f, \Omega]$ even if $\Omega$ is not a polytope. We begin with an overview of the method described in (Levin 1982). If we have a function $F$ such that

$$
\frac{\mathrm{d}}{\mathrm{d} x}\left[F(x) \mathrm{e}^{\mathrm{i} \omega g(x)}\right]=\left[F^{\prime}(x)+\mathrm{i} \omega g^{\prime}(x) F(x)\right] \mathrm{e}^{\mathrm{i} \omega g(x)}=f(x) \mathrm{e}^{\mathrm{i} \omega g(x)},
$$

then we can compute $I[f,(a, b)]$ trivially. Defining the differential operator $L[F]=F^{\prime}+\mathrm{i} \omega g^{\prime} F$ and rewriting the above equation as $L[F]=f$, we can now approximate $F$ by a function $v$ that is a linear combination of $\nu$ basis functions $\psi_{1}, \psi_{2}, \ldots, \psi_{\nu}$, using collocation with the operator $L$. In other words, we choose nodes $c_{1}<c_{2}<\cdots<c_{\nu}$, where $c_{1}$ and $c_{\nu}$ are the endpoints of the interval $\Omega$, and solve for $v$ using the system

$$
L[v]\left(c_{k}\right)=f\left(c_{k}\right), \quad k=1,2, \ldots, \nu .
$$

Olver $(2005 a)$ generalized this method in a manner similar to a Filon-type method, equipping collocation points with multiplicities $\boldsymbol{m} \in \mathbb{N}^{\nu}$. Now $v$ is a linear combination of $\tau=\mathbf{1}^{\top} \boldsymbol{m}-1$ functions. This results in a new system,

$$
\frac{\mathrm{d}^{i}}{\mathrm{~d} x^{i}} L[v]\left(c_{k}\right)=\frac{\mathrm{d}^{i}}{\mathrm{~d} x^{i}} f\left(c_{k}\right), \quad i=1,2, \ldots, m_{k}, \quad k=1,2, \ldots, \nu .
$$

We then define

$$
Q_{s}^{\mathrm{L}}[f,(a, b)]=v(b) \mathrm{e}^{\mathrm{i} \omega g(b)}-v(a) \mathrm{e}^{\mathrm{i} \omega g(a)},
$$

which is equivalent to $I[L[v]]$.

One huge benefit of Levin-type methods is that they work easily on complicated domains and complicated oscillators for which Filon-type methods utterly fail. We demonstrate the method on the quarter-circle $H=\{(x, y)$ : $\left.x^{2}+y^{2}<1, x, y>0\right\}$, however it works equally well on other domains that satisfy the nonresonance condition, including those in higher dimensions. In the univariate version we approximated $F$, where $L[F]=f$, which enabled us 
to 'push' the integral to the boundary of the interval, namely its endpoints. We use this idea as an inspiration for the multivariate case: we begin by determining an operator $L$ that will allow us to 'push' the integral to the boundary. To do so, we use differential forms along with the Stokes theorem. Suppose we have a function $F$ such that

$$
\int_{\partial H} F(x, y) \mathrm{e}^{\mathrm{i} \omega g(x, y)}(\mathrm{d} x+\mathrm{d} y)=\int_{H} f(x, y) \mathrm{e}^{\mathrm{i} \omega g(x, y)} \mathrm{d} V .
$$

Stokes' theorem tells us that

$$
\begin{aligned}
I[f] & =\int_{\partial H} F \mathrm{e}^{\mathrm{i} \omega g}(\mathrm{~d} x+\mathrm{d} y)=\int_{H} \mathrm{~d}\left[F \mathrm{e}^{\mathrm{i} \omega g}(\mathrm{~d} x+\mathrm{d} y)\right] \\
& =\int_{H}\left(F_{y}+\mathrm{i} \omega g_{y} F\right) \mathrm{e}^{\mathrm{i} \omega g} \mathrm{~d} y \wedge \mathrm{d} x+\left(F_{x}+\mathrm{i} \omega g_{x} F\right) \mathrm{e}^{\mathrm{i} \omega g} \mathrm{~d} x \wedge \mathrm{d} y \\
& =I\left[F_{x}+\mathrm{i} \omega g_{x} F-F_{y}-\mathrm{i} \omega g_{y} F\right]
\end{aligned}
$$

Hence we use the collocation operator $L[F]=F_{x}+\mathrm{i} \omega g_{x} F-F_{y}-\mathrm{i} \omega g_{y} F$. For simplicity, we write both the univariate and multivariate operator as $L[F]=$ $J[F]+\mathrm{i} \omega J[g] F$, where in two dimensions $J[F]=F_{x}-F_{y}$, and in one dimension $J[F]=F^{\prime}$. Thus we determine a linear combination of basis functions $v$ by solving the system

$$
\frac{\partial^{|\boldsymbol{i}|}}{\partial \boldsymbol{x}^{\boldsymbol{i}}} L[v]\left(\boldsymbol{c}_{k}\right)=\frac{\partial^{|\boldsymbol{i}|}}{\partial \boldsymbol{x}^{\boldsymbol{i}}} f\left(\boldsymbol{c}_{k}\right), \quad 0 \leq|\boldsymbol{i}| \leq m_{k}-1, \quad k=1,2, \ldots, \nu,
$$

where $\boldsymbol{c}_{1}, \ldots, \boldsymbol{c}_{\nu}$ is a sequence of nodes. Consequently,

$$
\begin{aligned}
I[f, H] \approx I[L[v], H]= & \int_{\partial H} v \mathrm{e}^{\mathrm{i} \omega g}(\mathrm{~d} x+\mathrm{d} y) \\
= & \int_{0}^{\frac{\pi}{2}}(\cos t-\sin t) v(\cos t, \sin t) \mathrm{e}^{\mathrm{i} \omega g(\cos t, \sin t)} \mathrm{d} t \\
& -\int_{0}^{1} v(0,1-t) \mathrm{e}^{\mathrm{i} \omega g(0,1-t)} \mathrm{d} t+\int_{0}^{1} v(t, 0) \mathrm{e}^{\mathrm{i} \omega g(t, 0)} \mathrm{d} t .
\end{aligned}
$$

We thus define $Q^{\mathrm{L}}[f, H]$ by approximating each of these univariate integrals using univariate Levin-type methods. For the proof of the asymptotic order we assume that the endpoints of each of these integrals have the same multiplicity as the associated vertex. For example, the multiplicity at $t=0$ of the first integral is the same as the multiplicity at $(\cos 0, \sin 0)=(1,0)$.

We will show that, as in a Filon-type method, $I[f, H]-Q^{\mathrm{L}}[f, H]=$ $\mathcal{O}\left(\omega^{-s-n}\right)=\mathcal{O}\left(\omega^{-s-2}\right)$, where $s$ is again the smallest vertex multiplicity. We begin by showing that $I[f, \Omega]-I[L[v], \Omega]=\mathcal{O}\left(\omega^{-s-n}\right)$, where $\Omega=H$ or a univariate interval. One might be tempted to prove this by considering it as a Filon-type method with $\phi=L[v]$. Indeed, it satisfies all the conditions of a Filon-type method, except for the fact that $L[v]$ depends on $\omega$. Hence, in 
order to prove the error, we also need to show that $f-L[v]$ and its derivatives are bounded for increasing $\omega$. To do so, we impose the regularity condition, which requires that the vectors $\boldsymbol{g}_{1}, \boldsymbol{g}_{2}, \ldots, \boldsymbol{g}_{\tau}$, where $\tau=\mathbf{1}^{\top} \boldsymbol{m}$-1, are linearly independent. Here

$$
\boldsymbol{g}_{j}=\left[\begin{array}{c}
\boldsymbol{\rho}_{j, 1} \\
\vdots \\
\boldsymbol{\rho}_{j, \nu}
\end{array}\right],
$$

where

$$
\boldsymbol{\rho}_{j, k}=\left[\begin{array}{c}
\frac{\partial^{\left|\boldsymbol{p}_{k, 1}\right|}}{\partial \boldsymbol{x}^{\boldsymbol{p}_{k, 1}}}\left(J[g] \psi_{j}\right)\left(c_{k}\right) \\
\vdots \\
\frac{\partial^{\left|\boldsymbol{p}_{k, n_{k}}\right|}}{\partial \boldsymbol{x}^{\boldsymbol{p}_{k, n_{k}}}}\left(J[g] \psi_{j}\right)\left(c_{k}\right)
\end{array}\right]
$$

while $\boldsymbol{p}_{k, 1}, \ldots, \boldsymbol{p}_{k, n_{k}} \in \mathbb{N}^{n}, n_{k}=\frac{1}{2} m_{k}\left(m_{k}+1\right)$, are all the vectors such that $\left|\boldsymbol{p}_{k, i}\right| \leq m_{k}-1$, lexicographically ordered.

Note that we can rewrite the system $(9)$ in the form $(P+\mathrm{i} \omega G) \boldsymbol{d}=\boldsymbol{f}$, where $G$ is the matrix whose $j$ th column is $\boldsymbol{g}_{j}, \mathrm{P}$ is a matrix independent of $\omega, \boldsymbol{d}$ is the vector of unknown coefficients in $v$, and $\boldsymbol{f}$ is defined as

$$
\boldsymbol{f}=\left[\begin{array}{c}
\boldsymbol{\sigma}_{1} \\
\vdots \\
\boldsymbol{\sigma}_{\tau}
\end{array}\right], \quad \boldsymbol{\sigma}_{k}=\left[\begin{array}{c}
\frac{\partial^{\left|\boldsymbol{p}_{k, 1}\right|}}{\partial \boldsymbol{x}^{\boldsymbol{p}_{k, 1}}} f\left(c_{k}\right) \\
\vdots \\
\frac{\partial^{\left|\boldsymbol{p}_{k, n_{k}}\right|}}{\partial \boldsymbol{x}^{\boldsymbol{p}_{k, n_{k}}}} f\left(c_{k}\right)
\end{array}\right], \quad k=1, \ldots, \nu .
$$

From Cramer's rule we know that $d_{k}=\operatorname{det} D_{k} / \operatorname{det}(P+\mathrm{i} \omega G)$, where $D_{k}$ is the matrix $P+\mathrm{i} \omega G$ with the $k$ th column replaced by $f$. Due to the regularity condition, $G$ is nonsingular, hence $[\operatorname{det}(P+\mathrm{i} \omega G)]^{-1}=\mathcal{O}\left(\omega^{-\tau}\right)$, where $\tau$ is equal to the number of rows in $G$. Moreover, it is clear that $\operatorname{det} D_{k}=\mathcal{O}\left(\omega^{\tau-1}\right)$. Hence $d_{k}=\mathcal{O}\left(\omega^{-1}\right)$, and $L[v]=\mathcal{O}(1)$ for increasing $\omega$. Thus, as in a Filontype method, $I[f, \Omega]-I[L[v], \Omega]=\mathcal{O}\left(\omega^{-s-n}\right)$.

If $\Omega$ is a univariate interval then we have just demonstrated that $I[f, \Omega]-$ $Q^{\mathrm{L}}[f, \Omega]=\mathcal{O}\left(\omega^{-s-1}\right)$. In the multivariate case (and sticking to our example of a quarter-circle: the general case is similar) we need to prove that $I[L[v], H]-$ $Q^{\mathrm{L}}[f, H]=\mathcal{O}\left(\omega^{-s-n}\right)$. Each of the integrands in (10) is of order $\mathcal{O}\left(\omega^{-1}\right)$. It follows that the approximations by $Q^{\mathrm{L}}$ are of order $\mathcal{O}\left(\omega^{-s-2}\right)$. Hence we have demonstrated that $I[f, H]-Q^{\mathrm{L}}[f, H]=\mathcal{O}\left(\omega^{-s-2}\right)$. It is clear that this proof can be generalized to other domains, with an asymptotic order $n+s$.

It should be emphasized that a Levin-type method attains exactly the same asymptotic order as a Filon-type method, using the same information about $f$. In fact, if $\Omega$ is a simplex and $g$ is a linear oscillator then the two methods are equivalent, assuming that the subintegrals in a Levin-type method have a sufficient number of data points (Olver 2005b). However, the latter requires 
significantly more operations, assuming that the computation of moments is efficient, since a system must be solved for each dimension. Moreover, (Olver $2005 a$ ) presents experimental evidence that suggests that Levin-type methods are typically less accurate than Filon-type methods, though this depends on the choice of oscillator $g$, on interpolation nodes, the closeness of $f$ to a polynomial and the choice of interpolation basis for the Levin-type method.

In Fig. 6, we approximate the same univariate integral as in Fig. 4, now with Levin-type methods in place of Filon-type methods. As can be seen, in conformity with the theory, the two methods share the same asymptotic order, while the Levin-type method exhibits somewhat lesser accuracy.
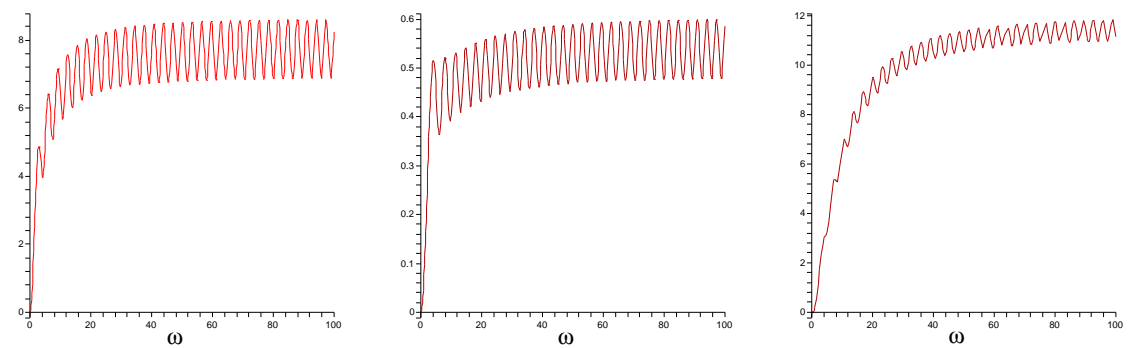

Fig. 6. Scaled error for $Q_{1}^{\mathrm{L}}$ with $\boldsymbol{c}=[-1,1], \boldsymbol{m}=[1,1]$ (on the left), $Q_{1}^{\mathrm{L}}$ with $\boldsymbol{c}=\left[-1,-\frac{3}{4}, \frac{3}{4}, 1\right], \boldsymbol{m}=[1,1,1,1]$ (at the centre) and $Q_{2}^{\mathrm{L}}$ with $\boldsymbol{c}=[-1,1], \boldsymbol{m}=[2,2]$ (on the right) for $\Omega=(-1,1), f(x)=x \sin x$ and $g(x)=x+\frac{1}{4} x^{2}$.

In Fig. 7 we see how well can a Levin-type method handle two-dimensional domains with nonlinear $g$. Specifically, we consider the quarter-circle $H=$ $\left\{(x, y): x^{2}+y^{2}<1, x, y>0\right\}$. In the first figure we collate at each vertex with multiplicity one for the bivariate system, and at the endpoints with multiplicity one for each univariate integral in (10). The second figure collocates with multiplicity two at each vertices and with multiplicity one at $\left(\frac{1}{3}, \frac{1}{3}\right)$, for the bivariate system, and collocates with just the endpoints with multiplicities two for each univariate system. Note that $H$, not being a polytope, represents a domain for which no viable theory exists for Filon-type methods.

In the univariate case it is possible to identify basis functions $\psi_{k}$ which lead to the highest-possible asymptotic order. Specifically, $\psi_{k}=f_{k+1} / g^{\prime}$, where the functions $f_{k}$ have already featured in the asymptotic expansion (4). We dwell no further on this issue, referring the reader to (Olver 2005a).

\section{Critical points}

Once $\nabla g$ is allowed to vanish in $\operatorname{cl} \Omega$, the asymptotic formula (5) is no longer valid. Worse, in a multivariate setting surprisingly little is known about 

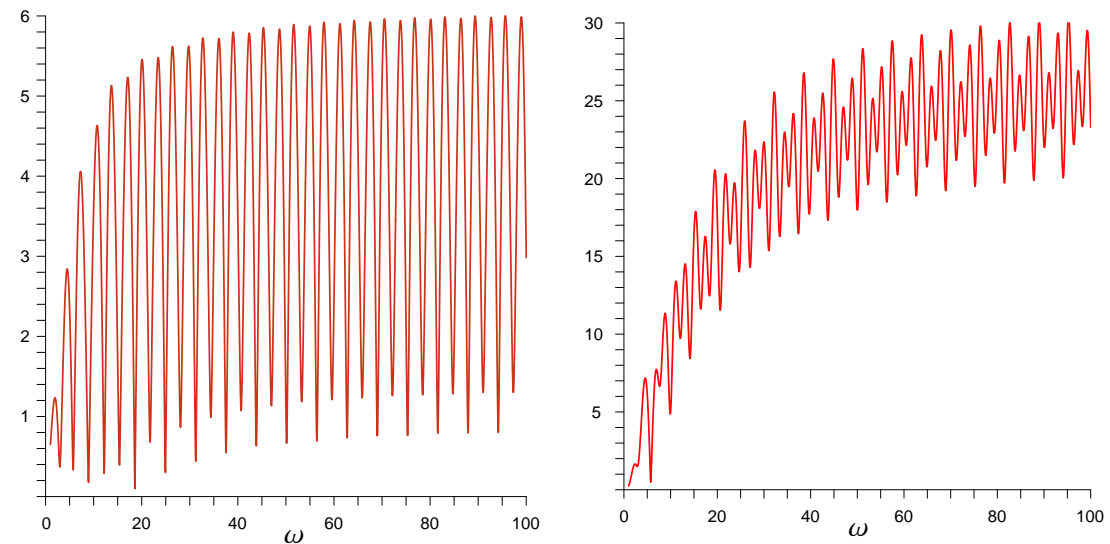

Fig. 7. Scaled error for $Q_{1}^{\mathrm{L}}$ (on the left) and $Q_{2}^{\mathrm{L}}$ (on the right) for $\Omega=H, f(x, y)=$ $\mathrm{e}^{x-2 y}$ and $g(x, y)=x^{3}+x-y$.

asymptotic expansions in the presence of high oscillation and critical points (Stein 1993). The situation is much clearer and better understood in a single dimension. ${ }^{4}$ This is due to the van der Corput theorem, which allows us to determine the asymptotic order of magnitude of (1) (Stein 1993). Moreover, the classical method of stationary phase provides an avenue of sorts, once we have taken care of the behaviour at the endpoints, toward an asymptotic expansion (Olver 1974, Stein 1993). Unfortunately, this technique falls short of providing the entire information required to construct an asymptotic expansion, while being complicated and cumbersome.

In this section we describe an alternative to the method of stationary phase which has been introduced in (Iserles \& Nørsett 2005a). We revisit the method of proof of Section 2, taking full advantage of the considerable simplification due to univariate setting. Let us suppose for simplicity that $\Omega=(a, b)$ and there exists a unique $\xi \in(a, b)$ such that $g^{\prime}(\xi)=0, g^{\prime \prime}(\xi) \neq 0$ and $g^{\prime}(x) \neq 0$ for $x \in[a, b] \backslash\{\xi\}$. Clearly, the assumption that there is just one critical point hardly represents loss of generality, since we can always partition $(a, b)$ into such subintervals. We will comment later on the case when also higher derivatives of $g$ vanish at $\xi$. Finally, the case of $\xi=a$ or $\xi=b$ can be obtained by fairly straightforward generalization of our technique and is left to the reader.

A single step of our expansion technique in the absence of critical points in a single dimension is

$$
I[f,(a, b)]=\frac{1}{\mathrm{i} \omega} \int_{a}^{b} \frac{f(x)}{g^{\prime}(x)} \frac{\mathrm{d}}{\mathrm{d} x} \mathrm{e}^{\mathrm{i} \omega g(x)} \mathrm{d} x
$$

\footnotetext{
${ }^{4}$ In the univariate case critical points are often termed "stationary points", but for consistency's sake we employ 'multivariate' terminology.
} 


$$
=\frac{1}{\mathrm{i} \omega}\left[\frac{\mathrm{e}^{\mathrm{i} \omega g(b)}}{g^{\prime}(b)} f(b)-\frac{\mathrm{e}^{\mathrm{i} \omega g(a)}}{g^{\prime}(a)} f(a)\right]-\frac{1}{\mathrm{i} \omega} I\left[\left(\frac{f}{g^{\prime}}\right)^{\prime},(a, b)\right]
$$

and it does not generalize to our setting since division by $g^{\prime}$ introduces polar singularity at $\xi$. Instead, we add and subtract $f(\xi)$ in the integrand,

$$
\begin{aligned}
I[f,(a, b)]= & f(\xi) \int_{a}^{b} \mathrm{e}^{\mathrm{i} \omega g(x)} \mathrm{d} x+\frac{1}{\mathrm{i} \omega} \int_{a}^{b} \frac{f(x)-f(\xi)}{g^{\prime}(x)} \frac{\mathrm{d}}{\mathrm{d} x} \mathrm{e}^{\mathrm{i} \omega g(x)} \mathrm{d} x \\
= & f(\xi) \mu_{0}(\omega)+\frac{1}{\mathrm{i} \omega}\left\{\frac{\mathrm{e}^{\mathrm{i} \omega g(b)}}{g^{\prime}(b)}[f(b)-f(\xi)]-\frac{\mathrm{e}^{\mathrm{i} \omega g(a)}}{g^{\prime}(a)}[f(a)-f(\xi)]\right\} \\
& -\frac{1}{\mathrm{i} \omega} I\left[\left(\frac{f-f(\xi)}{g^{\prime}}\right)^{\prime},(a, b)\right] .
\end{aligned}
$$

Note that $[f(x)-f(\xi)] / g^{\prime}(x)$ is a smooth function, since the singularity at $\xi$ is removable.

Iterating the last identity leads to an asymptotic expansion in the presence of a simple critical point. Thus, we define

$$
f_{0}(x)=f(x), \quad f_{m}(x)=\frac{\mathrm{d}}{\mathrm{d} x} \frac{f_{m-1}(x)-f_{m-1}(y)}{g^{\prime}(x)}, \quad m \in \mathbb{N},
$$

whence

$$
\begin{aligned}
& I[f,(a, b)] \sim \mu_{0}(\omega) \sum_{m=0}^{\infty} \frac{1}{(-\mathrm{i} \omega)^{m}} f_{m}(y) \\
& \quad-\sum_{m=0}^{\infty} \frac{1}{(-\mathrm{i} \omega)^{m+1}}\left\{\frac{\mathrm{e}^{\mathrm{i} \omega g(b)}}{g^{\prime}(b)}\left[f_{m}(b)-f_{m}(y)\right]-\frac{\mathrm{e}^{\mathrm{i} \omega g(a)}}{g^{\prime}(a)}\left[f_{m}(a)-f_{m}(y)\right]\right\} .
\end{aligned}
$$

For $x \neq \xi$ each $f_{m}$ is a linear combination of $f, f^{\prime}, \ldots, f^{(m)}$, but at $x=\xi$ we have

$$
\begin{aligned}
f_{0}(\xi)= & f(\xi) \\
f_{1}(\xi)= & \frac{1}{2} \frac{1}{g^{\prime \prime}(\xi)} f^{\prime \prime}(\xi)-\frac{1}{2} \frac{g^{\prime \prime \prime}(\xi)}{g^{\prime \prime 2}(\xi)} f^{\prime}(\xi) \\
f_{2}(\xi)= & \frac{1}{8} \frac{1}{g^{\prime \prime} 2(\xi)} f^{(\mathrm{iv})}(\xi)-\frac{5}{12} \frac{g^{\prime \prime \prime}(\xi)}{g^{\prime \prime 3}(\xi)} f^{\prime \prime \prime}(\xi)+\left[\frac{5}{8} \frac{g^{\prime \prime \prime}(\xi)}{g^{\prime \prime}(\xi)}-\frac{1}{4} \frac{g^{(\mathrm{iv})}(\xi)}{g^{\prime \prime 3}(\xi)}\right] f^{\prime \prime}(\xi) \\
& +\left[-\frac{5}{8} \frac{g^{\prime \prime \prime}(\xi)}{g^{\prime \prime 5}(\xi)}+\frac{2}{3} \frac{g^{(\mathrm{iv})}(\xi)}{g^{\prime \prime 4}(\xi)}-\frac{1}{8} \frac{g^{(\mathrm{v})}(\xi)}{g^{\prime \prime 3}(\xi)}\right] f^{\prime}(\xi)
\end{aligned}
$$

and so on: in general, each $f_{m}(\xi)$ is a linear combination of $f^{(i)}(\xi), i=$ $0,1, \ldots, 2 m$. The price tag of quadrature in the presence of critical point is the imperative to evaluate more derivatives there. 
Note that (12) is not a 'proper' asymptotic expansion, because of the presence of the function $\mu_{0}(\omega)$. In principle, it might have been possible to replace $\mu_{0}$ by its asymptotic expansion, e.g. using the method of stationary phase. This, however, is neither necessary nor, indeed, advisable. Assuming that $\mu_{0}$ can be computed - and we need this anyway for Filon-type methods! - it is best to leave it in place. According to the van der Corput theorem, $\mu_{0}(\omega) \sim \mathcal{O}\left(\omega^{-\frac{1}{2}}\right)$

It is straightforward to generalize our method of analysis to higher-order critical points. Thus, if $g^{(i)}(\xi)=0, i=1,2, \ldots, r, g^{(r+1)}(\xi) \neq 0$, in place of (11) we integrate by parts on the right in

$$
\begin{aligned}
I[f,(a, b)]= & \sum_{k=0}^{r-1} \frac{1}{k !} f^{(k)}(\xi) \int_{a}^{b}(x-\xi)^{k} \mathrm{e}^{\mathrm{i} \omega g(x)} \mathrm{d} x \\
& +\frac{1}{\mathrm{i} \omega} \int_{a}^{b} \frac{f(x)-\sum_{k=0}^{r-1} \frac{1}{k !} f^{(k)}(\xi)(x-\xi)^{k}}{g^{\prime}(x)} \frac{\mathrm{d}}{\mathrm{d} x} \mathrm{e}^{\mathrm{i} \omega g(x)} \mathrm{d} x .
\end{aligned}
$$

Again, we obtain removable singularity inside the integral. Note that by the van der Corput theorem $I[f,(a, b)]=\mathcal{O}\left(\omega^{-1 /(r+1)}\right)$.

Truncation of (12) results in an asymptotic method, a generalization of (7). Specifically,

$$
\begin{aligned}
Q_{s}^{\mathrm{A}}[f,(a, b)] & =\mu_{0}(\omega) \sum_{m=0}^{s-1} \frac{1}{(-\mathrm{i} \omega)^{m}} f_{m}(y) \\
& -\sum_{m=0}^{s-1} \frac{1}{(-\mathrm{i} \omega)^{m+1}}\left\{\frac{\mathrm{e}^{\mathrm{i} \omega g(b)}}{g^{\prime}(b)}\left[f_{m}(b)-f_{m}(y)\right]-\frac{\mathrm{e}^{\mathrm{i} \omega g(a)}}{g^{\prime}(a)}\left[f_{m}(a)-f_{m}(y)\right]\right\}
\end{aligned}
$$

bears asymptotic error of $s+\frac{1}{2}$.

Fig. 8 revisits the calculation from Section 1 that persuaded us in the inadequacy of Gaussian quadrature in the present setting: the calculation of $\int_{-1}^{1} \cos x \mathrm{e}^{\mathrm{i} \omega x^{2}} \mathrm{~d} x$. Note that $Q_{1}^{\mathrm{A}}$ requires just the values of $f$ at $-1,0,1$, while $Q_{2}^{\mathrm{A}}$ needs $f$ and $f^{\prime}$ at the endpoints and $f, f, f^{\prime \prime}$ at the critical point.

It is easy to generalize Filon-type methods to this setting. Nothing of essence changes. Thus, we choose nodes $a=c_{1}<c_{2}<\cdots<c_{\nu}=b$, taking care to include $\xi$ : thus, $c_{r}=\xi$ for some $r \in\{2, \ldots, \nu-1\}$. We interpolate to $f$ and its first $m_{k}-1$ derivatives at $c_{k}, k=1,2, \ldots, \nu$, with a polynomial $\varphi$ of degree $\boldsymbol{m}^{\top} \mathbf{1}-1$ and set

$$
Q_{s}^{\mathrm{F}}[f,(a, b)]=I[\varphi,(a, b)] .
$$

Here $s=\min \left\{m_{1},\left\lfloor\left(m_{r}-1\right) / 2\right\rfloor, m_{\mu}\right\}$. It follows at once from the asymptotic expansion that

$$
Q_{s}^{\mathrm{F}}[f,(a, b)]=I[f,(a, b)]+\mathcal{O}\left(\omega^{-s-\frac{1}{2}}\right), \quad|\omega| \gg 1,
$$



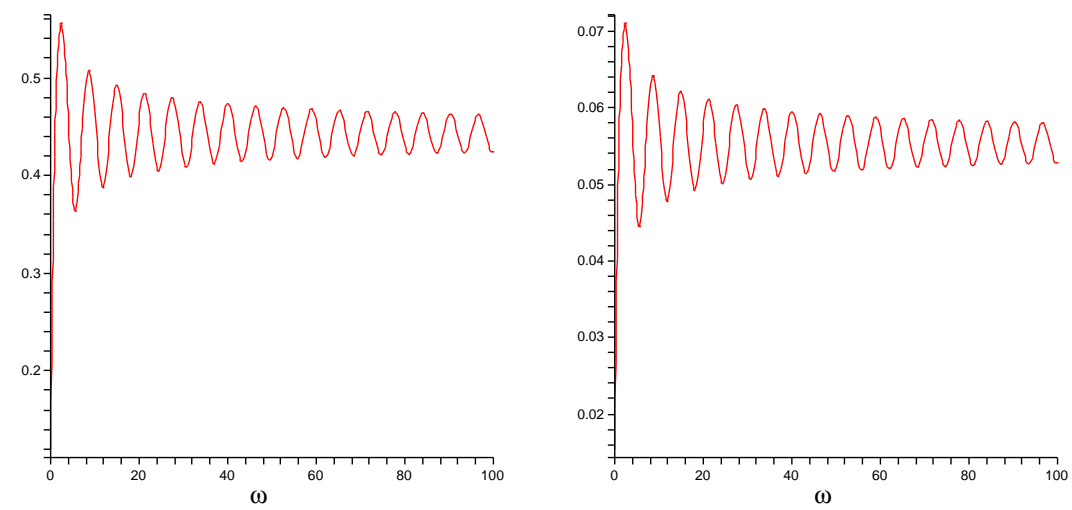

Fig. 8. The error for $Q_{1}^{\mathrm{A}}$ (on the left) and $Q_{2}^{\mathrm{A}}$ (on the right), scaled by $\omega^{\frac{3}{2}}$ and $\omega^{\frac{5}{2}}$ respectively, for $\Omega=(-1,1), f(x)=\cos x$ and $g(x)=x^{2}$, with a stationary point at the origin.

and the method is of asymptotic order $s+\frac{1}{2}$. As a matter of fact, a generalization of Filon in the presence of critical points is much more flexible than that of the asymptotic method. We can easily cater for any number of critical points, possibly of different degrees, once we include them among the nodes and choose sufficiently large multiplicities.

Fig. 9 shows the scaled error for three different Filon-type methods for the same problem as in Figs 1 and 8. Note how the accuracy greatly improves upon the addition of extra internal nodes. It is at present unclear why the addition of extra internal nodes has a much more dramatic effect in the presence of critical points.
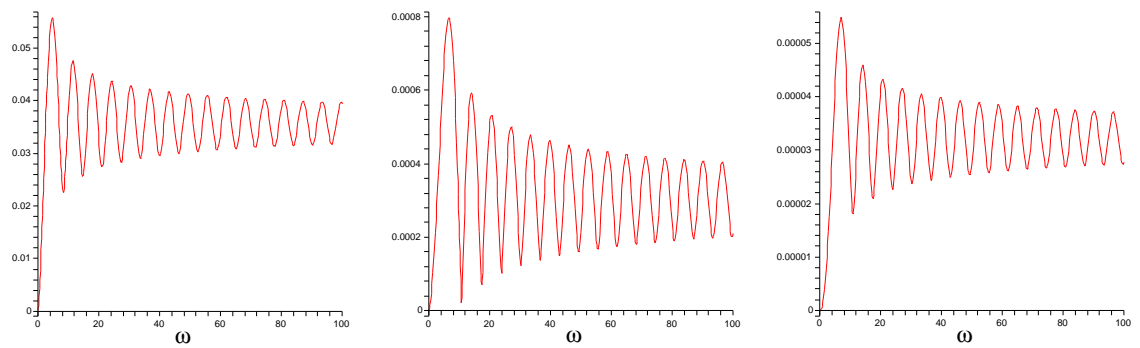

Fig. 9. Scaled error for $Q_{1}^{\mathrm{F}}$ with $\boldsymbol{c}=[-1,0,1], \boldsymbol{m}=[1,3,1]$ (on the left), $Q_{1}^{\mathrm{F}}$ with $\boldsymbol{c}=\left[-1,-\frac{1}{2}, 0, \frac{1}{2}, 1\right], \boldsymbol{m}=[1,1,3,1,1]$ (at the centre) and $Q_{2}^{\mathbf{A}}$ with $\boldsymbol{c}=[-1,0,1]$, $\boldsymbol{m}=[2,5,2]$ (on the right) for $\Omega=(-1,1), f(x)=\cos x$ and $g(x)=x^{2}$. 


\section{Conclusions and pointers for further research}

The first and foremost lesson to be drawn from our analysis is that, once we can understand the mathematics of high oscillation, we gain access to a wide variety of effective and affordable algorithms. This, of course, is a truism that we might apply to just about every issue in mathematical computation, yet it is of particular importance in the current framework. The overwhelming wisdom in much of classical treatment of rapidly oscillating phenomena is to find means to make high oscillation go away. Thus, the 'rule of a thumb', ubiquitous in signal processing, that a function should be sampled sufficiently often within each period: in the current setting this translates to an approximation of $I[f,(a, b)]$, say, by partitioning $(a, b)$ into a very large number of subintervals of length $\mathcal{O}\left(\omega^{-1}\right)$ and using Gaussian quadrature within each 'panel'. However, the conclusion of this paper, and also of much contemporary work in the discretization of highly oscillatory ordinary differential equations, is that high oscillation renders solution easier!

Another reason why it is important to emphasize the role of mathematical understanding in our endeavour is that so little is known about the asymptotics of $I[f, \Omega]$ in general domains $\Omega$. A fairly complete theory exists for $\Omega=\mathbb{R}^{n}$ and for $\Omega=\mathbb{S}^{n-1}$ (the $(n-1)$-sphere), at least as long as there are no critical points (Stein 1993). Yet, once we concern ourselves with bounded domains with boundary and allow for the presence of critical points, a great deal remains to be done. It is a sobering thought that the asymptotic behaviour of $I[f, \Omega]$, where $\Omega \subset \mathbb{R}^{2}$ is bounded and with piecewise-smooth boundary, is unknown in general even if there are no critical points! Clearly, it depends on the geometry of $\partial \Omega$, an issue to which we will return, but it is presently unclear how.

A thread running through our entire analysis is the centrality of an asymptotic expansion of $I[f, \Omega]$. Once (5) is available, its truncation presents us with an immediate means to compute the integral. Moreover, even if the explicit form of (5) is unavailable, the very existence and known structure of an asymptotic formula allow us to analyse better and more flexible quadrature methods.

The assumption that $\Omega$ is a polytope is very restrictive. A naive means of a generalization to arbitrary bounded domains $\Omega$ with piecewise-smooth boundary is to approximate it from within by a convergent sequence of polytopes and use the dominating convergence theorem. This, however, might fall foul of the nonresonance condition. Consider, for example, the linear oscillator $g(\boldsymbol{x})=\boldsymbol{\kappa}^{\top} \boldsymbol{x}, \boldsymbol{x} \in \mathbb{R}^{2}$ and a circular wedge $\Omega$ with angle $\alpha$,

$$
\Omega=\left\{\boldsymbol{x} \in \mathbb{R}^{2}: x_{1}^{2}+x_{2}^{2}<1, \arctan \frac{x_{2}}{x_{1}}<\alpha\right\} .
$$

As long as

$$
\pm \frac{1}{\sqrt{\kappa_{1}^{2}+\kappa_{2}^{2}}}\left[\begin{array}{c}
-\kappa_{2} \\
\kappa_{1}
\end{array}\right] \notin \partial \Omega
$$


we can approximate $\Omega$ with narrow wedges, pass to a limit and obtain an asymptotic expansion, expressing $I[f, \Omega]$ in terms of $f$ and its derivatives at $(0,0)$ and $(\cos \alpha, \pm \sin \alpha)$. Yet, if the above condition fails, the nonresonance condition must be breached upon passage to the limit. It is important to make it clear that the fault is definitely not in our method of proof. Once the resonance condition fails, an (5)-like expansion is no longer true! For simplicity, consider the bivariate unit disc $\Omega=\mathbb{S}^{1}$ and, again, a linear oscillator. We have

$$
I\left[f, \mathbb{S}^{1}\right]=\int_{-1}^{1} \int_{-\left(1-x^{2}\right)^{\frac{1}{2}}}^{\left(1-x^{2}\right)^{\frac{1}{2}}} f(x, y) \mathrm{e}^{\mathrm{i} \omega\left(\kappa_{1} x+\kappa_{2} y\right)} \mathrm{d} y \mathrm{~d} x .
$$

Expanding asymptotically in the inner interval similarly to (4), we thus have (assuming for simplicity that $\kappa_{2} \neq 0$ )

$$
\begin{aligned}
& I\left[f, \mathbb{S}^{1}\right] \sim-\frac{1}{\kappa_{2}} \int_{-1}^{1} \sum_{m=0}^{\infty} \frac{1}{(-\mathrm{i} \omega)^{m+1}}\left[\mathrm{e}^{\mathrm{i} \omega \kappa_{2}\left(1-x^{2}\right)^{\frac{1}{2}}} \frac{\mathrm{d}^{m}}{\mathrm{~d} y^{m}} f\left(x,\left(1-x^{2}\right)^{\frac{1}{2}}\right)\right. \\
& \left.-\mathrm{e}^{-\mathrm{i} \omega \kappa_{2}\left(1-x^{2}\right)^{\frac{1}{2}}} \frac{\mathrm{d}^{m}}{\mathrm{~d} y^{m}} f\left(x,-\left(1-x^{2}\right)^{\frac{1}{2}}\right)\right] \mathrm{e}^{\mathrm{i} \omega \kappa_{1} x} \mathrm{~d} x \\
& =-\frac{1}{\kappa_{2}} \sum_{m=0}^{\infty} \frac{1}{(-\mathrm{i} \omega)^{m+1}} \int_{-1}^{1} \frac{\mathrm{d}^{m}}{\mathrm{~d} y^{m}} f\left(x,\left(1-x^{2}\right)^{\frac{1}{2}}\right) \mathrm{e}^{\mathrm{i} \omega\left[\kappa_{1} x+\kappa_{2}\left(1-x^{2}\right)^{\frac{1}{2}}\right]} \mathrm{d} x \\
& +\frac{1}{\kappa_{2}} \sum_{m=0}^{\infty} \frac{1}{(-\mathrm{i} \omega)^{m+1}} \int_{-1}^{1} \frac{\mathrm{d}^{m}}{\mathrm{~d} y^{m}} f\left(x,-\left(1-x^{2}\right)^{\frac{1}{2}}\right) \mathrm{e}^{\mathrm{i} \omega\left[\kappa_{1} x-\kappa_{2}\left(1-x^{2}\right)^{\frac{1}{2}}\right]} \mathrm{d} x,
\end{aligned}
$$

an infinite sum of univariate integrals. However, before we rush to expand them asymptotically, we observe that the new oscillators have critical points at $\pm \kappa_{1} /\left(\kappa_{1}^{2}+\kappa_{2}^{2}\right)^{\frac{1}{2}}$. Our immediate conclusion is that $I\left[f, \mathbb{S}^{1}\right] \sim \mathcal{O}\left(\omega^{-\frac{3}{2}}\right)$, rather than the $\mathcal{O}\left(\omega^{-2}\right)$ which we might have expected. Worse, all our three approaches fail. The moments of $g(x)=\kappa_{1} x \pm \kappa_{2}\left(1-x^{2}\right)^{\frac{1}{2}}$ are unknown, hence we have neither an asymptotic expansion á la (12) nor a Filon-type method. Moreover, a Levin-type method fails because of the presence of critical points.

As long as the nonresonance condition is maintained throughout the approximation of $\Omega$ by polytopes, our methods can be extended to this setting. This has been already done for Levin-type methods in (Olver 2005b): cf. the discussion leading to Fig. 7 in Section 3.

Our narrative underlies the importance of further research into quadrature methods for highly oscillatory integrals, in particular in the presence of critical points and when exact moments are unavailable. There are a few natural ways forward, in particular Filon-type methods with suitable approximate moments and Levin-type methods with special treatment of small neighbourhoods surrounding critical points (where the integral does not oscillate rapidly). Both approaches are under active consideration. Another option is quadrature methods based on altogether new principles, e.g. the recent technique of Huybrechs \& Vandewalle (2005), who approximate (1) in a single 
dimension using a complex-valued path along which $\mathrm{e}^{\mathrm{i} \omega g(x)}$ does not oscillate. The underlying idea there, assuming that both $f$ and $g$ can be analytically extended to the complex plane, is to find a path from each endpoint of $\Omega=(a, b)$ to infinity alongside which $g(z)-g(a)$ and $g(z)-g(b)$, respectively, are real and negative. In place of (1) it is then possible to integrate from $b$ to $z=\infty$ and then from $\infty$ to $a$. Because of exponential decay of the integrand, each integration can be accomplished by familiar Gauss-Laguerre quadrature and the outcome matches Filon-type and Levin-type methods in its asymptotic behaviour. We further note that in the presence of critical points there is a need to integrate also along paths joining them with $z=\infty$ in a fairly nontrivial manner.

Other challenges in highly oscillatory quadrature abound. One obvious generalization of (1) is

$$
\int_{\Omega} f(\boldsymbol{x}) K(\omega, \boldsymbol{x}) \mathrm{d} V
$$

where $K$ oscillates rapidly for $|\omega| \gg 1$. Filon-type methods have been generalized to this setting in the important special case of the Bessel oscillator, when $\Omega=(a, b)$ and $K(\omega, x)=\mathrm{J}_{\nu}(\omega x)$ (Xiang 2005) but, by and large, this is an uncharted territory. Another terra incognita is (1) where $\Omega$ is a general bounded manifold with boundary, immersed in $\mathbb{R}^{n}$.

We have already touched upon applications of highly oscillatory quadrature to numerical methods for rapidly oscillating differential equations. Even more ambitious goal is the analysis of highly oscillatory Fredholm equations of the second kind

$$
\int_{a}^{b} f(x, \omega) K(x, y, \omega) \mathrm{d} x=\lambda(\omega) f(y, \omega)-g(y), \quad y \in[a, b],
$$

where $\lambda(\omega) \in \mathbb{C}$ is not an eigenvalue of the underlying operator, and of the corresponding spectral problem

$$
\int_{-1}^{1} \varphi(x, \omega) K(x, y, \omega)=\lambda(\omega) \varphi(y, \omega), \quad y \in[a, b] .
$$

Both (14) and (15) are highly interesting because of their applications in electromagnetics and in laser theory, but their treatment by 'our' methods is hampered by the fact that the function $f$ in (14) and the eigenfunction $\varphi$ in (15) themselves oscillate. This renders integration by parts, along the lines of Section 2, fairly useless.

The spectral problem (15) has been solved for the kernel $K(x, y, \omega)=$ $\mathrm{e}^{\mathrm{i} \omega x y}$, by demonstrating that $\varphi$ obeys a specific Sturm-Liouville problem (Cochran \& Hinds 1974). The asymptotic behaviour of the spectrum for $K(x, y, \omega)=\mathrm{e}^{\mathrm{i} \omega|x-y|}$ has been investigated by Ursell (1969). A detailed investigation of this kernel, inclusive of an asymptotic expansion of both eigenvalues and the solution of (14) in negative powers of $\omega$ will feature in a forthcoming paper by Brunner, Iserles and Nørsett. Yet, in their full generality, highly 
oscillatory integral equations of this kind represent an enduring and difficult challenge.

\section{References}

Cochran, J. A. \& Hinds, E. W. (1974), 'Eigensystems associated with the complexsymmetric kernels of laser theory', SIAM J. Appld Maths 26, 776-786.

Degani, I. \& Schiff, J. (2003), RCMS: Right correction Magnus series approach for integration of linear ordinary differential equations with highly oscillatory terms, Technical report, Weizmann Institute of Science.

Filon, L. N. G. (1928), 'On a quadrature formula for trigonometric integrals', Proc. Royal Soc. Edinburgh 49, 38-47.

Flinn, E. A. (1960), 'A modification of Filon's method of numerical integration', $J$ ACM 7, 181-184.

Huybrechs, D. \& Vandewalle, S. (2005), On the evalution of highly oscillatory integrals by analytic continuation, Technical report, Katholieke Universiteit Leuven,.

Iserles, A. (2002), 'On the global error of discretization methods for highly-oscillatory ordinary differential equations', BIT 42, 561-599.

Iserles, A. (2004), 'On the method of Neumann series for highly oscillatory equations', BIT 44, 473-488.

Iserles, A. \& Nørsett, S. P. (2005a), 'Efficient quadrature of highly oscillatory integrals using derivatives', Proc. Royal Soc. A 461, 1383-1399.

Iserles, A. \& Nørsett, S. P. (2005b), 'On quadrature methods for highly oscillatory integrals and their implementation', BIT. To appear.

Iserles, A. \& Nørsett, S. P. (2006), 'Quadrature methods for multivariate highly oscillatory integrals using derivatives', Maths Comp. To appear.

Levin, D. (1982), 'Procedure for computing one- and two-dimensional integrals of functions with rapid irregular oscillations', Maths Comp. 38, 531-538.

Lorenz, K., Jahnke, T. \& Lubich, C. (2005), 'Adiabatic integrators for highly oscillatory second order linear differential equations with time-varying eigendecomposition', BIT 45, 91-115.

Luke, Y. L. (1954), 'On the computation of oscillatory integrals', Proc. Cambridge Phil. Soc. 50, 269-277.

Munkres, J. R. (1991), Analysis on Manifolds, Addison-Wesley, Reading, MA.

Olver, F. W. J. (1974), Asymptotics and Special Functions, Academic Press, New York.

Olver, S. (2005a), 'Moment-free numerical integration of highly oscillatory functions', IMA J. Num. Anal. To appear.

Olver, S. (2005b), Multivariate Levin-type method, Technical Report TBD, DAMTP, University of Cambridge.

Stein, E. (1993), Harmonic Analysis: Real-Variable Methods, Orthogonality, and Oscillatory Integrals, Princeton University Press, Princeton, NJ.

Ursell, F. (1969), 'Integral equations with a rapidly oscillating kernel', J. London Math. Soc. 44, 449-459.

Xiang, S. (2005), 'On quadrature of Bessel transformations', J. Comput. Appld Maths 177, 231-239. 